\title{
Polarization-Insensitive Electroabsorptive Modulation Using Interdiffused InGaAs(P)-InP Quantum Wells
}

\author{
Wallace C. H. Choy, E. Herbert Li, Senior Member, IEEE, and Joseph Micallef, Member, IEEE
}

\begin{abstract}
This is a theoretical study to demonstrate the use of interdiffusion in the realization of polarization insensitivity at the band-edge. Two InGaAs-InP quantum well as-grown structures have been investigated: one with lattice-matched condition and the other with small as-grown tensile strain $(0.15 \%)$. The interdiffusion is considered to take place on the Group V (As and P) sublattice only. As a result, a tensile strain is produced which merges the heavy- and light-hole states in order to achieve polarization insensitivity. Criteria to develop polarization-insensitive quantum wells (QW's) using interdiffusion are presented here. When the two-phase interdiffusion mechanism is modeled, the results show that the well barrier interfaces of the $Q W$ maintain an abrupt profile while the well width remains constant after interdiffusion. The two interdiffused QW structures considered here can produce polarization insensitive electroabsorption at operation wavelengths around $1.55 \mu \mathrm{m}$. The one with latticematched condition is particularly attractive since it only requires an easy (high-yield) fabrication process with a simple postprocessing thermal annealing to achieve polarization insensitivity.
\end{abstract}

Index Terms-Diffusion process, electrooptic modulation, optical polarization, quantum-confined Stark effect, quantum-well interdiffusion, quantum-well intermixing, quantum wells, strain layered material.

\section{INTRODUCTION}

$\mathbf{I}$ $\mathrm{N}$ THE realization of monolithic photonic integration, quantum wells (QW's) can provide a large absorption change for use in optical switching and modulation [1] and yet be able to integrate with lasers and detectors. However, these QW structures exhibit various drawbacks including a strong polarization dependence of the incoming optical fields (transverse electric (TE) and transverse magnetic (TM) polarization) [2]. Optical signals traveling a relatively long distance in optical fibers usually do not preserve the polarization of light, so that in an electroabsorption modulator, polarization dependence is undesirable. In order to remove this barrier, several polarization-independent or polarizationinsensitive QW material systems have been proposed for electroabsorptive devices [3]-[8]. Among these QW's, there

Manuscript received August 14, 1996; revised April 4, 1997. This work was supported by a Hong Kong RGC grant and a University of Hong Kong CRCG grant. The work of W. C. H. Choy was supported by the Sir Edward Youde Memorial Fellowship.

W. C. H. Choy is with the University of Surrey, U.K.

E. H. Li is with the Department of Electrical and Electronic Engineering, University of Hong Kong, Pokfulam Road, Hong Kong.

J. Micallef is with the Department of Microelectronics, University of Malta, Msida MSD 06, Malta.

Publisher Item Identifier S 0018-9197(97)05451-1. are basically three types of structures: quasi-parabolic QW structures [6], tensile strain QW structures [3]-[5], [8], and a combination of these two [7]. All of them can produce similar electroabsorptive changes for both TE and TM optical polarization of light. In this paper, we propose a fourth type which depends on the interdiffusion-induced tensile strain in the QW's.

Conventional polarization-insensitive $\operatorname{InGaAs}(\mathrm{P})-\mathrm{InP}$ QW structures which operate at photon wavelengths of $\sim 1.55 \mu \mathrm{m}$, normally have as-grown well widths between 11 and $12 \mathrm{~nm}$, with the as-grown tensile strains being $\sim 0.3 \%$ [4], [9]. We propose here the use of QW interdiffusion [10], in order to generate the tensile strain in an $\operatorname{InGaAs}(\mathrm{P})-\mathrm{InP}$ QW structure required for obtaining polarization insensitivity. Since a large portion of the tensile strain is produced by interdiffusion, only a small strain is required in an as-grown QW with well width of $11 \mathrm{~nm}$. More interestingly, the lattice-matched condition is sufficient for an as-grown QW with a well width of $12 \mathrm{~nm}$ prior to interdiffusion. The difficulties in growing highly strained as-grown QW's can thus be bypassed. In addition, by using such a simple and controllable interdiffusion technology, the operation wavelength of the modulator can be adjusted because of the modifiable QW bandgap energy through interdiffusion [11], [12]. All the above features make the interdiffused QW (DFQW) an attractive structure for developing polarizationinsensitive modulators. It should be noted that the interdiffused InGaAs-InP QW material has already been studied in some detail [11], [13], [14] and it has been successfully realized in various optical devices, such as waveguides [12] and lasers [15].

In this paper, we will theoretically study the effects of interdiffusion of two InGaAs(P)-InP DFQW's on the electroabsorptive polarization insensitivity: one QW structure having a well width of $11 \mathrm{~nm}$ and $0.15 \%$ as-grown tensile strain, and the other one grown lattice-matched, with a 12-nm well width. In Section II, the model in obtaining the DFQW absorption coefficient is discussed. In Section III, the criteria and steps to achieve electroabsorptive polarization insensitivity are presented. Section IV presents the results which include discussions on the advantage of Group $\mathrm{V}$ only interdiffusion for the development of polarization insensitivity; proposals of the polarization insensitive electroabsorptive change of DFQW's with and without as-grown tensile strain in the well layer are also presented. In Section VI, conclusions will be drawn. 


\section{Model of THE DFQW Optical Parameters}

In this section, the effect of interdiffusion on the subbands of the QW's are briefly discussed, which is followed by their corresponding absorption coefficient, $\alpha(\omega)$, and their change of absorption coefficient, $\Delta \alpha(\omega)$, induced by an externally applied electric field.

There are three types of interdiffusion processes in InGaAs-InP QWs: 1) Group III (In, Ga atoms) only interdiffusion [16]-[18]; 2) Group V (As, P) only interdiffusion [19], [20]; and 3) interdiffusion on both Group III and V sublattices [18], [21]. Only Group III interdiffusion induces compressive strain in the well and tensile strain in the barriers, while only Group V interdiffusion induces tensile strain in the well and compressive strain in the barriers. Interdiffusion on the two sublattices can induce either tensile or compressive strains in the well or it can maintain a lattice-matched structure. In order to induce a tensile strain large enough to realize polarization insensitivity, interdiffusion on the Group V sublattice only is used here. During the interdiffusion, not only is strain induced in the DFQW, but the fundamental transition energy also increases. This implies that the operation wavelength can be adjusted. One model which has been proposed for Group V only interdiffusion is a two-phase interdiffusion mechanism with different diffusion coefficients in the barrier and the well layers [20], [22]. This implies a "discontinuity" of the Group V concentration profile at the interface across the QW structure after interdiffusion. For a QW heterostructure, where the InGaAs well is sandwiched between two InP barriers, the interdiffusion process can be represented by a set of diffusion equations, respectively, for different species. This is defined as follows:

$$
\frac{\partial C_{i}(z, t)}{\partial t}=D_{i} \frac{\partial^{2} C_{i}(z, t)}{\partial z^{2}}
$$

where $z$ is the growth direction, $t$ is the annealing time, the $i$ subscript denotes the barrier for $|z| \geq L$ or the well for $|z|<L . L$ is the position of the QW interface from the well center $(z=0) ; C$ and $D$ are the concentration of the diffusion species, and their diffusion coefficients, respectively. In solving this partial differential equation, two continuity conditions must be satisfied at the well/barrier interfaces (i.e., $z= \pm L)$, namely

$$
\begin{gathered}
\left.C_{\text {barrier }}(t)\right|_{z= \pm L}=\left.k C_{\text {well }}(t)\right|_{z= \pm L} \\
\left.D_{\text {barrier }} \frac{\partial C_{\text {barrier }}(t)}{\partial z}\right|_{z= \pm L}=\left.D_{\text {well }} \frac{\partial C_{\text {well }}(t)}{\partial z}\right|_{z= \pm L} .
\end{gathered}
$$

Equation (2) models the discontinuous $(k \neq 1)$ concentration at the interface while (3) expresses the flux continuity. Further details have been thoroughly discussed in [22]. Equation (1) is solved by using a finite difference method to obtain the concentration distribution of the diffused species after an annealing time $t$. This concentration ratio gives the spatial molar fraction (composition) of the interdiffused As concentration $\tilde{y}$ in the $\operatorname{In}_{x_{o}} \mathrm{Ga}_{1-x_{o}} \mathrm{As}_{\tilde{y}} \mathrm{P}_{1-\tilde{y}}-\mathrm{InP} \mathrm{QW}$ structure. The notation $x_{0}$ is the concentration of the In atoms (Group III composition) and since the interdiffusion only involves Group $\mathrm{V}$ composition, $x_{0}$ is a fixed constant. The induced tensile strain strongly depends on the extent of interdiffusion. It should be noted that in order to use an as-grown latticematched $\operatorname{In}_{x} \mathrm{Ga}_{1-x} \mathrm{As}_{y} \mathrm{P}_{1-y}$-InP system as a candidate to achieve polarization insensitivity, the relation $y=2.2(1-$ $x$ ) should be used for the as-grown condition [23]. The various stages of interdiffusion can be obtained by varying the annealing time and temperature, where the latter affects the diffusion coefficients.

The interdiffusion-induced quantum-confinement profiles and the electron and hole subband edges are calculated numerically [24]. The field effect is calculated according to a scheme developed by Bloss [25]. The subband envelope functions for the conduction band $\chi_{C}$, valence band $\chi_{V}$, and their transition energy $E_{C V}$ are first calculated. These subbands are then used to calculate the heavy-hole $(\mathrm{HH})$ and light-hole (LH) related $1 S$ exciton bound states (binding energies and wavefunctions) by a perturbation variation method [26].

The bound states absorption coefficient is given as

$$
\begin{aligned}
\alpha_{\text {bound }}(\omega)= & \frac{e^{2} \mu_{\|}^{*} \omega}{6 \varepsilon_{0} c_{0} n_{r} m_{C}^{*} E_{C V}^{2} L_{z}} \\
& \cdot M_{O} \sum_{C, V}\left|\left\langle\chi_{C} \mid \chi_{V}\right\rangle\right|^{2} I_{C V}(\hbar \omega)
\end{aligned}
$$

where

$$
\begin{aligned}
M_{0} & =\frac{E_{g}\left(E_{g}+\Delta_{0}\right)}{E_{g}+\frac{2}{3} \Delta_{0}} \\
I_{C V}(\hbar \omega) & =\int_{0}^{\infty} \wp(E) S(E) L(E) d E
\end{aligned}
$$

$\left|\left\langle\chi_{C} \mid \chi_{V}\right\rangle\right|^{2}$ is the probability of the wavefunction overlapping between the $\chi_{C}$ and the $\chi_{V}, \varepsilon_{0}$ is the permittivity in free space, $c_{0}$ is the velocity of light in free space, $n_{r}$ is the refractive index, $e$ is electronic charge, $m_{c}^{*}$ is the effective electron mass, and $\mu_{/ /}^{*}$ is the electron-hole reduced effective mass. In (4b), $E_{g}$ is the interdiffusion modified bandgap at $z=0$ and $\Delta_{O}$ is the diffused spin-orbit splitting gap. In (4c)

$$
L(E)=\frac{\Gamma_{B}}{\pi\left\{\left[E_{C V}+E-\hbar \omega\right)^{2}+\Gamma_{B}^{2}\right\}}
$$

$S(E)$ is the Sommerfield enhancement factor and is assumed to be unity, $L(E)$ is the Lorentzian broadening factor, and $\wp(E)$ is the polarization factor. For light propagating along the quantum layer, both the TE and TM polarizations exist and the polarization factors are given by $\gamma_{0}^{\mathrm{TE}}=\frac{3}{4}\left(1+E_{R}\right)$ for $\mathrm{HH}, \frac{5}{4}\left[1-\frac{3}{5} E_{R}\right]$ for $\mathrm{LH},{ }_{\gamma} \mathrm{TM}=\frac{3}{2}\left(1-E_{R}\right)$ for $\mathrm{HH}$, and $\frac{1}{2}\left(1+3 E_{R}\right)$ for LH where $E_{R}=\left(E_{C}+E_{H}\right) /\left(E_{C}+E_{H}+E\right)$. In (4d), $\Gamma_{B}$ is the bound state linewidth (half-width halfmaximum) broadening factor, $E_{C V}=E_{g}+E_{C}+E_{H}$. The exciton absorption coefficient, $\alpha_{\operatorname{exc}}(\omega)$, is given by

$$
\alpha_{\text {exc }}(\omega)=\frac{A \omega}{c_{0} n_{r}}|\chi(\rho=0)|^{2} \frac{\Gamma_{X B}}{\pi\left\{\left(E_{\mathrm{exc}}-\hbar \omega\right)^{2}+\Gamma_{X B}^{2}\right\}}
$$

where

$$
\begin{aligned}
A & =\frac{e^{2} \hbar^{2}}{3 \varepsilon_{0} m_{C}^{*} E_{C V}^{2} L_{z}} M_{0}\left|\left\langle\chi_{C 1} \mid \chi_{V 1}\right\rangle\right|^{2} \wp \\
E_{\text {exc }} & =E_{C 1}+E_{H 1}+E_{g}+E_{b}
\end{aligned}
$$


is the excitonic transition energy and $\Gamma_{X B}$ is the exciton linewidth (half-width half-maximum) broadening factor. For the $1 S$ exciton, only $\rho=0$ is allowed and hence $\wp^{\mathrm{TE}}=\frac{3}{2}$ $(\mathrm{HH}), \frac{1}{2}(\mathrm{LH})$, and $\wp^{\mathrm{TM}}=0(\mathrm{HH}), 2(\mathrm{LH})$. The total absorption coefficient $\alpha(\omega)$ is given by $\alpha(\omega)=\alpha_{\text {bound }}(\omega)+$ $\alpha_{\text {exc }}(\omega)$. The change of absorption coefficient $\Delta \alpha(\omega)$ is obtained using

$$
\Delta \alpha(\omega)=\alpha_{F \neq 0}(\omega)-\alpha_{F=0}(\omega) .
$$

The contrast ratio (CR) for an electroabsorptive modulator is defined as the relative optical intensity modulation and is given as

$$
\mathrm{CR}(\mathrm{dB})=10 \log \left[\frac{\exp \left(-\alpha_{\mathrm{ON}} l\right)}{\exp \left(-\alpha_{\mathrm{OFF}} l\right)}\right]
$$

where $\alpha_{\mathrm{ON}}$ and $\alpha_{\mathrm{OFF}}$ are the absorption in the on and off state, respectively, and $l$ is the modulation length. Here, the optical confinement factor is assumed to be unity.

\section{CRITERION AND StePS TO DEVEloP DFQW POLARIZATION INSENSITIVITY}

The absorption coefficient of a conventional rectangular QW is polarization dependent. One of the reasons for this dependence is the splitting of $\mathrm{HH}$ and $\mathrm{LH}$ states at the band edge. In the following, we will present the criteria and develop the design steps to achieve polarization insensitivity by using Group V interdiffusion in InGaAs-InP QW's. To achieve the electroabsorptive polarization insensitivity, the first criterion is to generate an adequate amount of shear strain which can at least counterbalance the splitting of $\mathrm{HH}$ and $\mathrm{LH}$ states due to the quantum confinement [8], i.e.,

$$
2 E_{s} \leq E_{\mathrm{LH}}-E_{\mathrm{HH}}
$$

where $E_{s}$ is the shear deformation potential, and $E_{\mathrm{LH}}$ and $E_{\mathrm{HH}}$ are the heavy-hole and light-hole energy levels, respectively. $E_{s}$ is multiplied by 2 because an approximately equivalent amount of shear strain influences both $\mathrm{HH}$ and $\mathrm{LH}$ while under the assumption that the spin-orbit splitting has a relatively small influence on the LH-related strain. Instead of using an asgrown InGaAs-InP QW with 0.3\%-0.7\% tensile strain [8], [9], we propose to use Group $\mathrm{V}$ interdiffusion to produce enough tensile strain such that (8) can be satisfied.

To maintain the DFQW active cavity under a coherent pseudomorphic condition is a crucial criterion in developing the polarization-insensitive modulators. During interdiffusion, the effective critical layer thickness of the DFQW continues to vary since the induced strain and the Group V compositions vary in the DFQW system. The QW may therefore degrade into a structure with dislocations. From our results, the maximum induced strain in the two DFQW structures considered here with well widths of 11 and $12 \mathrm{~nm}$ are $0.45 \%$ (after $0.4 \mathrm{~h}$ of $750^{\circ} \mathrm{C}$ annealing) and $0.32 \%$ (after 1 $\mathrm{h}$ of $750^{\circ} \mathrm{C}$ annealing), respectively. These DFQW's should be experimentally achievable without any dislocations since wider DFQW's with an as-grown well width of $20 \mathrm{~nm}$ (exceeding the critical thickness) and at longer annealing time $(2 \mathrm{~h})$ under the same annealing temperature have been produced [22].

There are various parameters that can modify the tensile strain in the $\operatorname{InGaAs}(\mathrm{P})-\mathrm{InP} \mathrm{DFQW}$. The as-grown composition itself can generate tensile strain in the well layer. A reduction of the as-grown indium concentration from the $y=$ $2.2(1-x)$ condition in the well layer introduces tensile strain. After interdiffusion, the alloy concentration, the well width, diffusion temperature, and time all contribute to the resulting strain in the QW system. This tensile strain can merge the $\mathrm{HH}$ and $\mathrm{LH}$ states and result in polarization insensitivity. The higher the diffusion temperature and the longer the diffusion time producing the interdiffusion on the Group V sublattice, the larger is the tensile strain that results in the well.

The amount of as-grown $\mathrm{P}$ content in the well layer is crucial to the development of polarization-insensitive DFQW's. When an amount $(1-y)$ of $\mathrm{P}$ is added to the as-grown latticematched $\mathrm{In}_{x} \mathrm{Ga}_{1-x} \mathrm{As}_{y} \mathrm{P}_{1-y}-\mathrm{InP} \mathrm{QW}$, the $\mathrm{C}-\mathrm{HH}$ (first electron and first heavy hole) and $\mathrm{C}-\mathrm{LH}$ (first electron and first light hole) transition energies increase (blue shift), and the splitting between the $\mathrm{LH}$ and $\mathrm{HH}$ also increases. Taking an as-grown lattice-matched QW with $L_{z}=11 \mathrm{~nm}$ as an example, when the $\mathrm{P}$ content in the well increases from $0-0.1$ under latticematched conditions, C-HH (C-LH) blue shifts from 0.7958 $(0.8166)$ to $0.8238 \mathrm{eV}(0.8490 \mathrm{eV})$. At the same time, the energy difference between $\mathrm{HH}$ and $\mathrm{LH}$ states increases from 0.0209 to $0.0252 \mathrm{eV}$ (difference of $4 \mathrm{meV}$ ). In Group V interdiffusion, since $\mathrm{P}$ diffuses from barrier to well and As diffuses from well to barrier, the transition energies increase as shown in Fig. 1. The longer the annealing time, the larger the transition energies. Since tensile strain in the well increases with annealing time, the difference of the transition energies (splitting of $\mathrm{HH}$ and $\mathrm{LH}$ ) reduces. As annealing time reaches $0.4 \mathrm{~h}, \mathrm{HH}$ and $\mathrm{LH}$ transition energies merge, hence polarization insensitivity is obtained. However, starting with an as-grown quaternary well (i.e., InGaAsP) creates two disadvantages for the interdiffusion to produce polarization insensitivity. The first one is that with the $\mathrm{P}$ content, the splitting between $\mathrm{HH}$ and $\mathrm{LH}$ increases, implying that larger tensile strains are required to bring back the $\mathrm{LH}$ and $\mathrm{HH}$ states together. Thus longer annealing time is required to generate stronger tensile strain. However, if a low initial as-grown tensile strain is introduced in an as-grown rectangular QW structure, only a small tensile strain is required to be produced from interdiffusion. The second disadvantage is that the increase of as-grown $\mathrm{P}$ content in the well will increase the transition energy of the as-grown QW structure, thereby reducing the adjustable range of transition energy by interdiffusion. This reduces the tuning ability of the DFQW to reach the target wavelength of $1.55 \mu \mathrm{m}$. Therefore, a material system with a small as-grown tensile strain in the InGaAs-InP QW without any as-grown $\mathrm{P}$ in the well is also considered here in addition to the lattice-matched $\mathrm{QW}$.

Chronological design steps for achieving electroabsorptive polarization insensitivity can now be drawn. The first step is to remove the effect of splitting between the $\mathrm{LH}$ and $\mathrm{HH}$ states. This implies that appropriate diffusion temperature and diffusion time should be selected. The second step is to select 
the concentration of indium and the well width of the as-grown QW structure in order to produce the operation wavelength within the range of $1.52-1.56 \mu \mathrm{m}$, which is of interest for current optical erbium-doped fiber amplifier technology. As a final step, it should be ensured that the DFQW structure remains coherently strained. These three steps are mutually related because the interdiffusion will reduce the operation wavelength while the concentration of indium will affect the splitting of $\mathrm{HH}$ and LH states. The resulting DFQW's may not satisfy the coherent pseudomorphic condition and results would have to be checked experimentally.

\section{RESUlts AND Discussions}

The two DFQW structures studied here are an undoped $\mathrm{In}_{0.51} \mathrm{Ga}_{0.49} \mathrm{As}-\mathrm{InP} \mathrm{QW}$ with well width of $11 \mathrm{~nm}$ and $0.15 \%$ as-grown tensile strain, as well as a lattice-matched undoped $\mathrm{In}_{0.53} \mathrm{Ga}_{0.47} \mathrm{As}-\mathrm{InP} \mathrm{QW}$ with well width of $12 \mathrm{~nm}$. Both of them are considered to be annealed under the same diffusion temperature of $750^{\circ} \mathrm{C}$. The diffusion coefficient of the well and that of the barrier, as well as the concentration ratio are taken from a typical structure [22]. The exciton broadening factors of $\mathrm{HH}$ and $\mathrm{LH}$ are considered to be the same and with a value of $\Gamma_{X B}=10 \mathrm{meV}$. The electroabsorptive modulation operates at $F=0$ and $100 \mathrm{kV} / \mathrm{cm}$. The $\mathrm{ON}$ state is designed at $F=0$. The operation wavelength is selected at the dip of the biased exciton absorption at which $\Delta \alpha_{\mathrm{TE}}$ equals $\Delta \alpha_{\mathrm{TM}}$ in order to obtain polarization insensitivity. Before discussing the absorption change of the DFQW's, the implications of using the two-phase model are discussed.

\section{A. Implications of Using the Two-Phase Model for Group V Interdiffusion}

Applying the two-phase interdiffusion model, the potential profile of the DFQW, shown in Fig. 2(a) (dotted line), exhibits approximately the same shape as the as-grown profile (solid line) with only a slight deviation at the bottom of the well. The diffused well width equals the as-grown well width because the model entails a discontinuous interface for the Group $\mathrm{V}$ composition even after interdiffusion. This is defined by (1) and (2), and thus, as shown in Fig. 2(b), the $\mathrm{P}$ profile after interdiffusion (dotted line) remains similar to its asgrown profile (solid line). As a consequence, a confinement potential profile Fig. 2(a) (dotted line) with an abrupt interface is obtained and its width equals that of the as-grown QW. It should be noted that when the compositional profile after interdiffusion in InGaAs-InP is modeled by an error function distribution both an abrupt confinement profile (in the case of interdiffusion on the Group III sublattice only) [24] and a graded confinement profile (in the case of interdiffusion on the two sublattices) [27] can result. In the latter case, shown in Fig. 2(a) (dashed line), it is clearly difficult to determine the effective well width.

A constant well width with interdiffusion would simplify the development of a polarization-insensitive modulator; a graded profile makes it difficult to determine parameters in (4) and (5) such as the effective well width $L_{z}$ and the broadening factors $\Gamma_{B}, \Gamma_{X B}$. Interdiffusion as a result of the

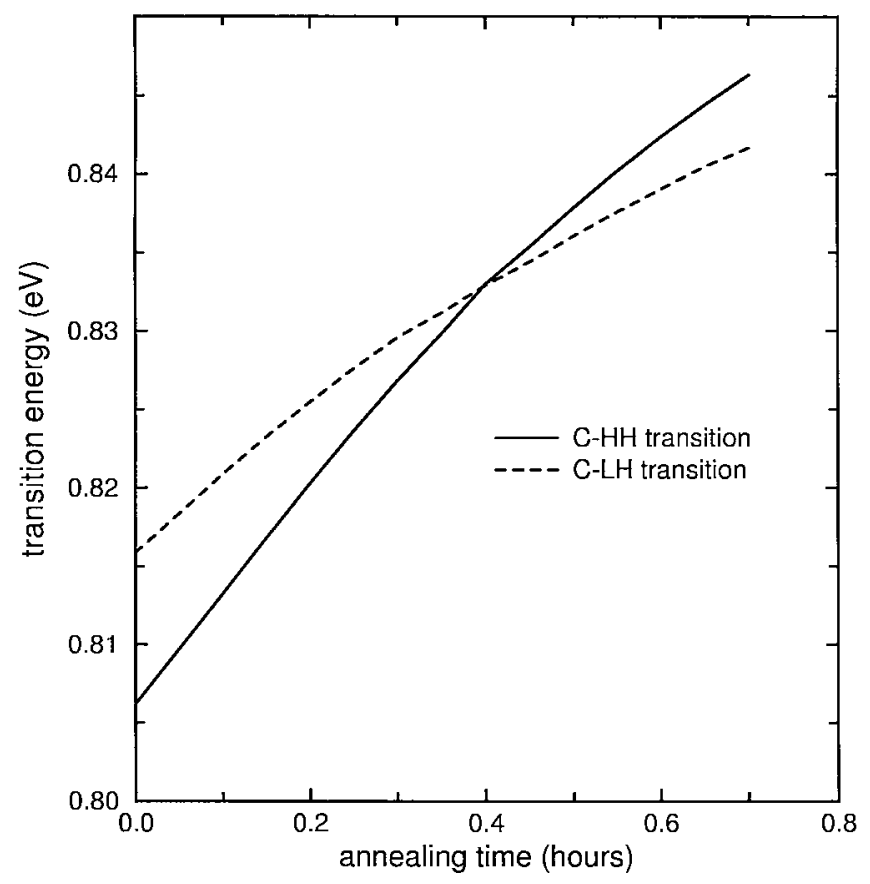

Fig. 1. The change of $\mathrm{C}-\mathrm{HH}$ (solid line) and $\mathrm{C}-\mathrm{LH}$ (dash line) transition energies of DFQW (with In content of 0.51 , well width $11 \mathrm{~nm}$ ) with increasing annealing time.

two-phase mechanism would maintain the same well width, so that the effect of well-width variation would be reduced, thus improving the potential for the tailoring of the polarization insensitivity. Suitable tensile strain in InGaAs-InP can also be produced in the well, in the case of interdiffusion at different rates on the two sublattices, resulting in the merging of the $\mathrm{HH}$ and $\mathrm{LH}$ transition energies [28]. In this case, the confinement energy exhibits a graded profile with the consequent complications in determining parameters such as width $L_{z}, \Gamma_{B}, \Gamma_{X B}$.

\section{B. Polarization-Insensitive Electroabsorption Change}

For the case of $x=0.51$, the $\mathrm{C}-\mathrm{HH}$ and $\mathrm{C}-\mathrm{LH}$ exciton transition energies can successfully merge together when annealing time increases to $0.4 \mathrm{~h}$, as shown in Fig. 1. The TE and TM absorption coefficient spectra of the DFQW with this annealing time are shown in Fig. 3. The $\mathrm{C}-\mathrm{HH}$ and $\mathrm{C}-\mathrm{LH}$ exciton absorption edges in the TE polarization overlap at $F=0$, verifying the merging of $\mathrm{HH}$ and $\mathrm{LH}$ states. It can be seen that the TE and TM absorption spectra overlap over a range of photon wavelengths for both $F=0$ and 100 $\mathrm{kV} / \mathrm{cm}$, showing that polarization-insensitive electroabsorption is possible. The operation region can be found from the absorption change spectra, as shown in Fig. 4, from which it can be observed that the operation wavelength locates between 1.54 and $1.56 \mu \mathrm{m}$ with a maximum polarization-insensitive absorption change of $\sim 1200 \mathrm{~cm}^{-1}$. Fig. 5 shows the CR in $\mathrm{dB}$ of the DFQW electroabsorptive modulator with modulation interaction length of $50 \mu \mathrm{m}$. For this DFQW cavity, assuming the optical confinement is unity, a maximum CR of $18 \mathrm{~dB}$ is obtained at photon wavelength of $1.542 \mu \mathrm{m}$. The attractive feature here is that the amount of tensile strain is increased 


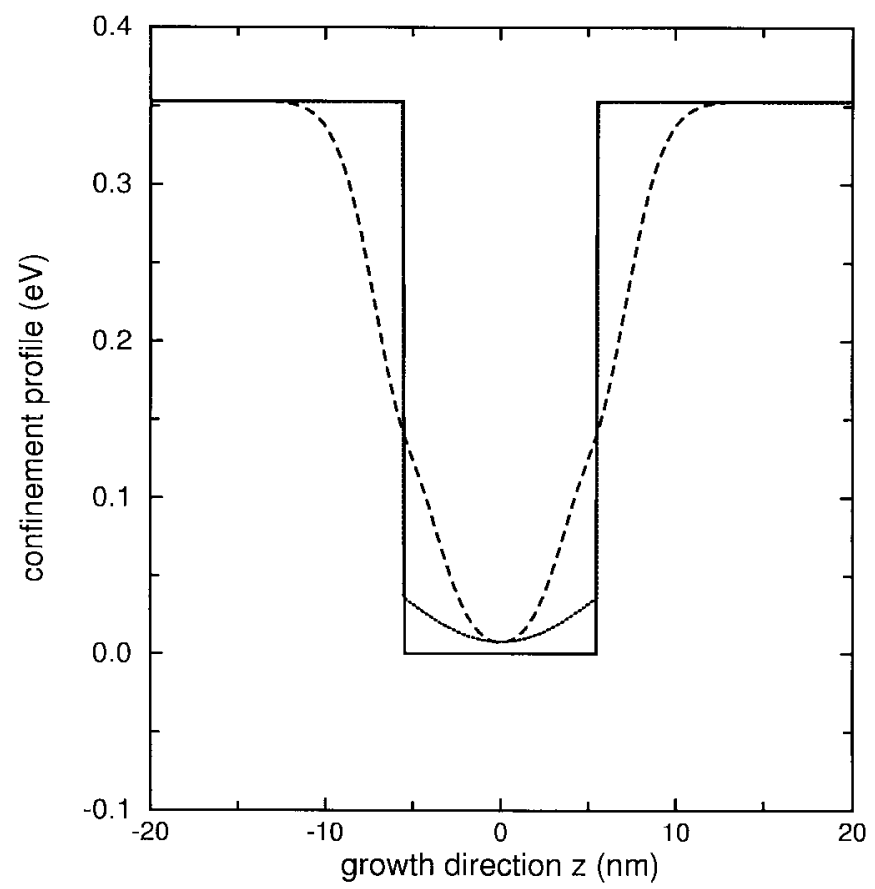

(a)

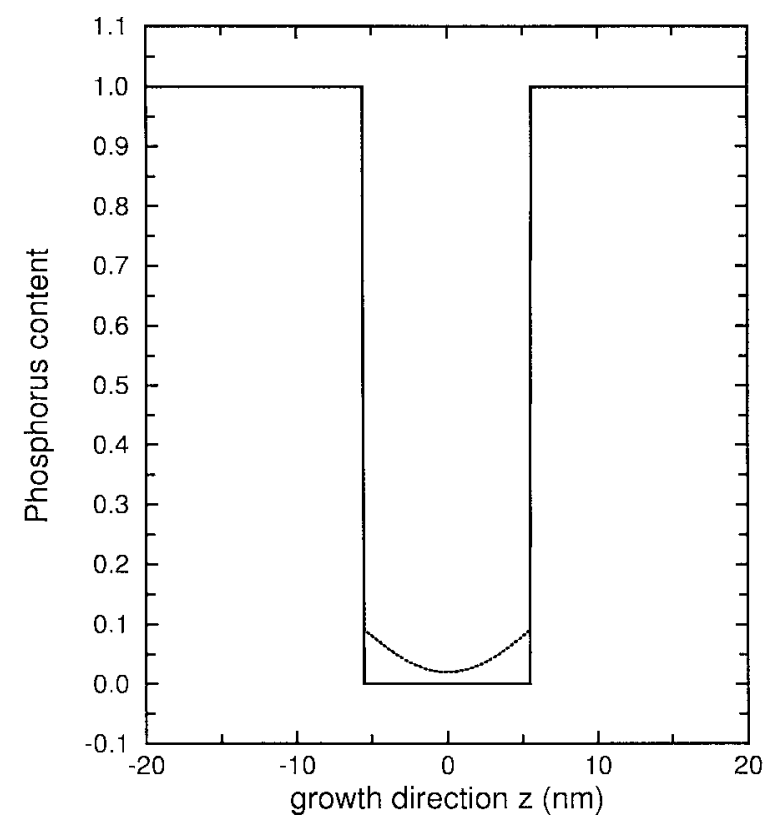

(b)

Fig. 2. (a) Electron potential profile of InGaAs-InP QW with well width of $11 \mathrm{~nm}$ and In content of 0.51: 1) square QW (solid line), 2) DFQW after Group V two phase interdiffusion with annealing time $0.4 \mathrm{~h}$ (dotted line), and 3) DFQW after interdiffusion on both Group III and Group V sublattices (dashed line). (b) Phosphorus profile of as-grown QW (solid line) and DFQW after Group V interdiffusion (dotted line).

by more than $200 \%$ in the DFQW (as shown in Fig. 6) as compared with its as-grown tensile strain. The average tensile strain is $0.36 \%$ and the maximum tensile strain positions at the interfaces with a value of $0.46 \%$ when the as-grown tensile strain is only $0.15 \%$. This means that a smaller asgrown tensile strain $(0.15 \%) \mathrm{QW}$ can produce polarization insensitivity after interdiffusion and thus ease the material growth and the device fabrication.

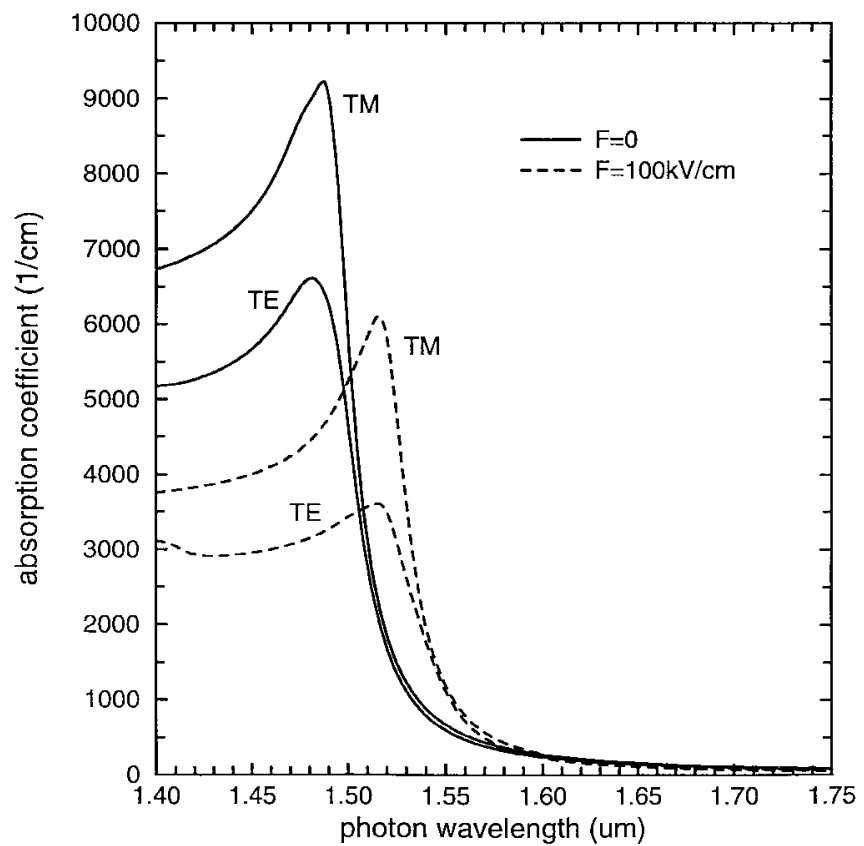

Fig. 3. The TE and TM absorption coefficient spectra of DFQW with In content of 0.51 , well width $11 \mathrm{~nm}$, and annealing of $0.4 \mathrm{~h}$ : (a) applied field $F=0$ (solid line) and (b) $F=100 \mathrm{kV} / \mathrm{cm}$ (dashed line).

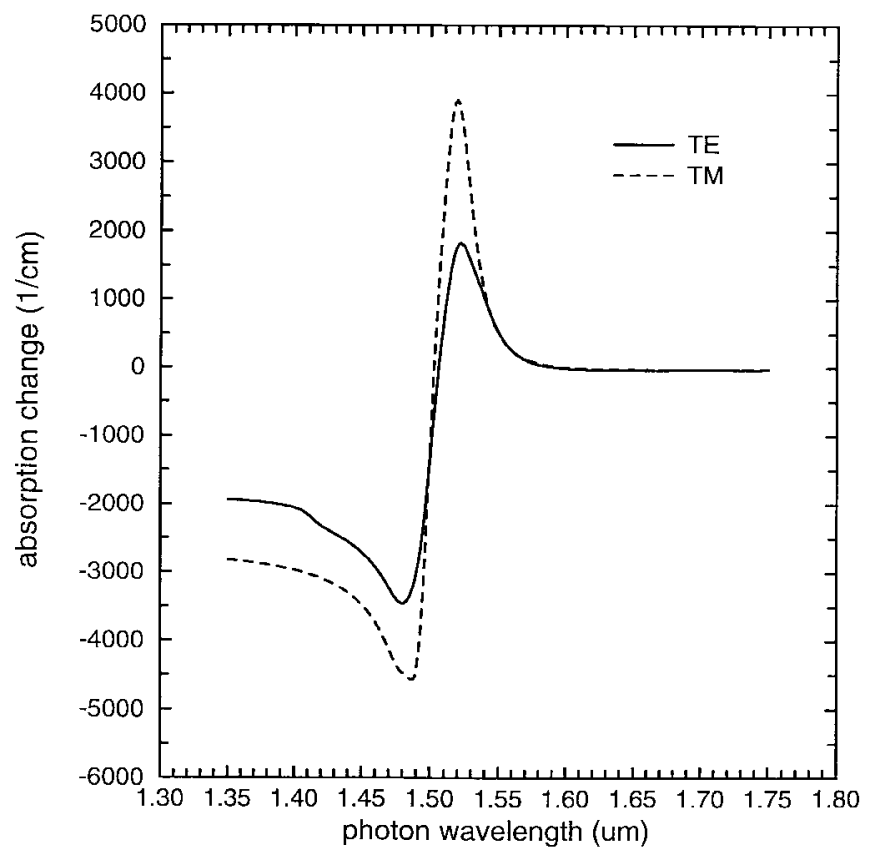

Fig. 4. The TE and TM absorption change spectra of DFQW with In content of 0.51 , well width $11 \mathrm{~nm}$, and annealing of $0.4 \mathrm{~h}$ with $F=100 \mathrm{kV} / \mathrm{cm}$.

In another structure with lattice-matched condition $(x=$ 0.53 ), electroabsorptive polarization insensitivity can be achieved after one hour of annealing. The TE and TM absorption spectra are shown in Fig. 7. The maximum absorption change of $\sim 1000 \mathrm{~cm}^{-1}$ (CR of $\sim 16 \mathrm{~dB}$ ) can be obtained at photon wavelength of $1.55 \mu \mathrm{m}$ as shown in Fig. 8. In this structure, the maximum strain at interfaces is $0.32 \%$ and the average strain is $0.27 \%$. As compared to the experimental structure with the same well width $(12 \mathrm{~nm})$ [4], 


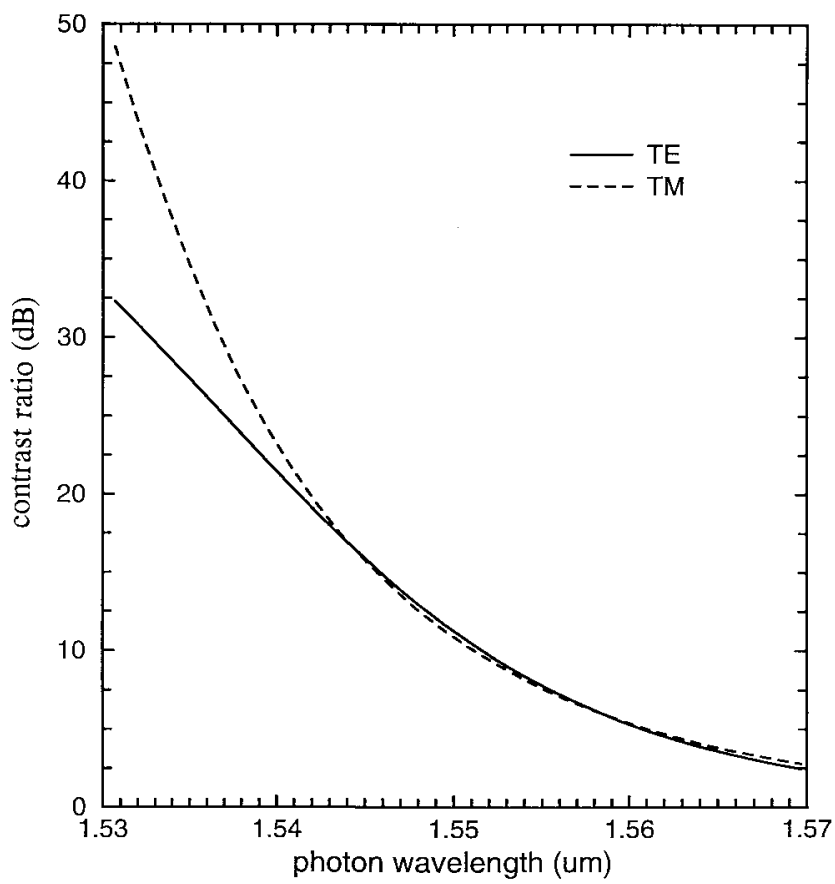

Fig. 5. The CR in $\mathrm{dB}$ of a full DFQW (the one with $x=0.51$ ) electroabsorptive modulator with length $50 \mu \mathrm{m}$.

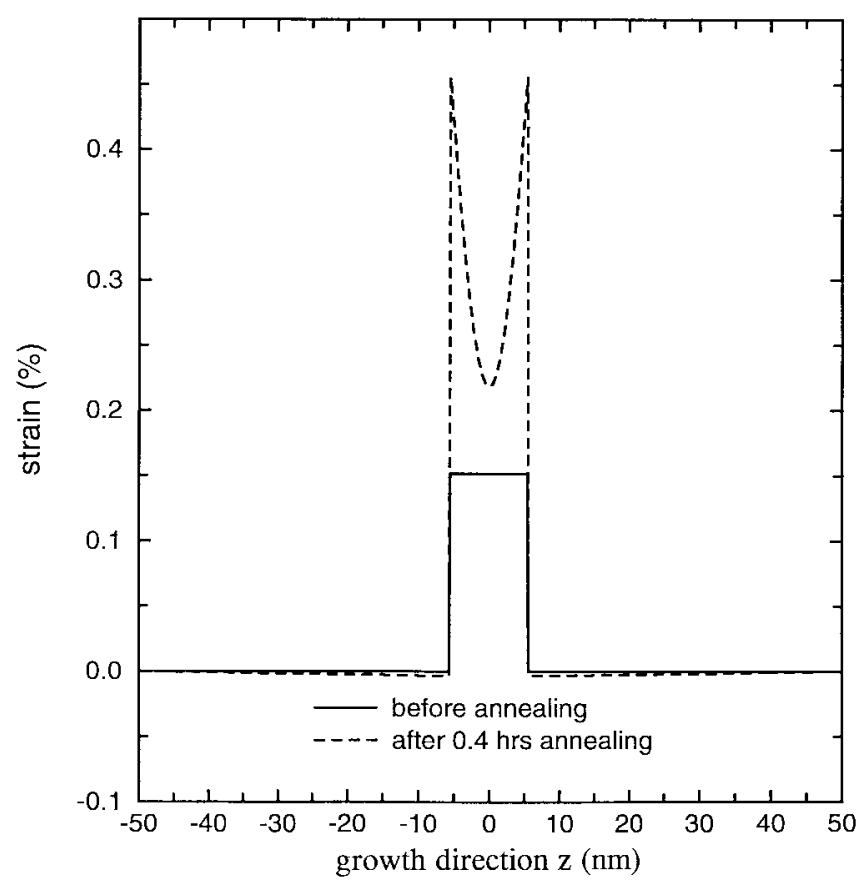

Fig. 6. The strain profile of InGaAs-InP QW with well width of $11 \mathrm{~nm}$ and In content of 0.51: (a) square QW (solid line) and (b) DFQW after annealing time of $0.4 \mathrm{~h}$ (dashed line).

where a $0.3 \%$ as-grown strained $\mathrm{In}_{0.49} \mathrm{Ga}_{0.51} \mathrm{As}-\mathrm{InP}$ square QW was fabricated, we here start with a lattice-matched asgrown QW, and polarization insensitivity can be obtained at around the $1.55-\mu \mathrm{m}$ wavelength.

\section{CONCLUSION}

We have investigated the effect of interdiffusion on the electroabsorptive polarization insensitivity in $\operatorname{InGaAs}(\mathrm{P})-\mathrm{InP}$

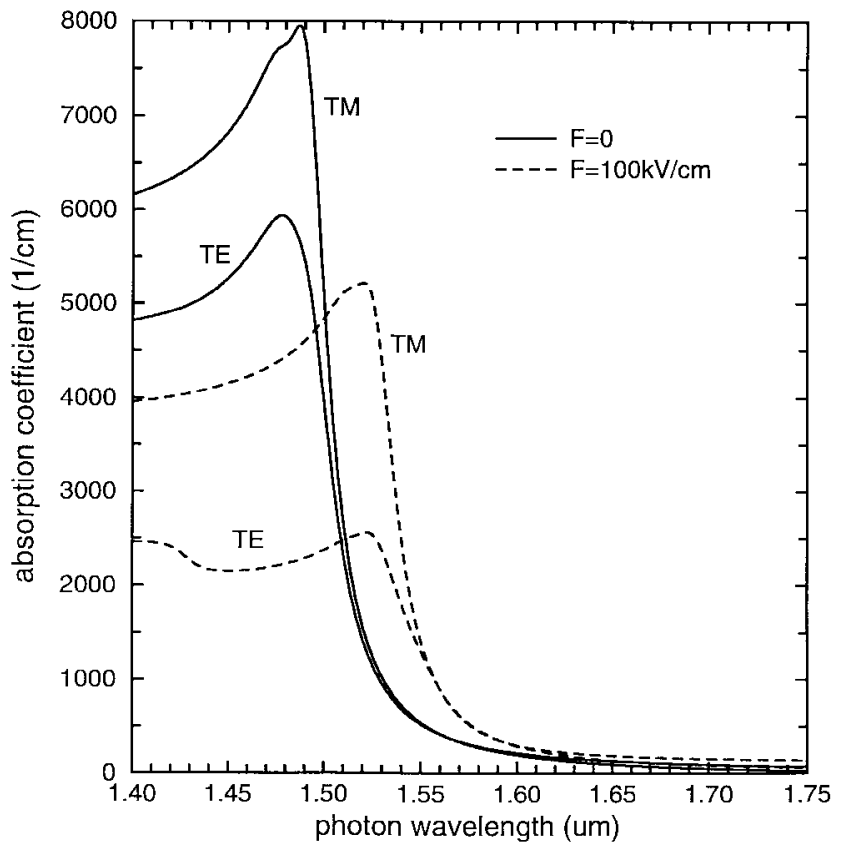

Fig. 7. The TE and TM absorption coefficient spectra of DFQW with In content of 0.53 , well width $12 \mathrm{~nm}$, and annealing of $1 \mathrm{~h}$ : (a) applied field $F=0$ (solid line) and (b) $F=100 \mathrm{kV} / \mathrm{cm}$ (dash line).

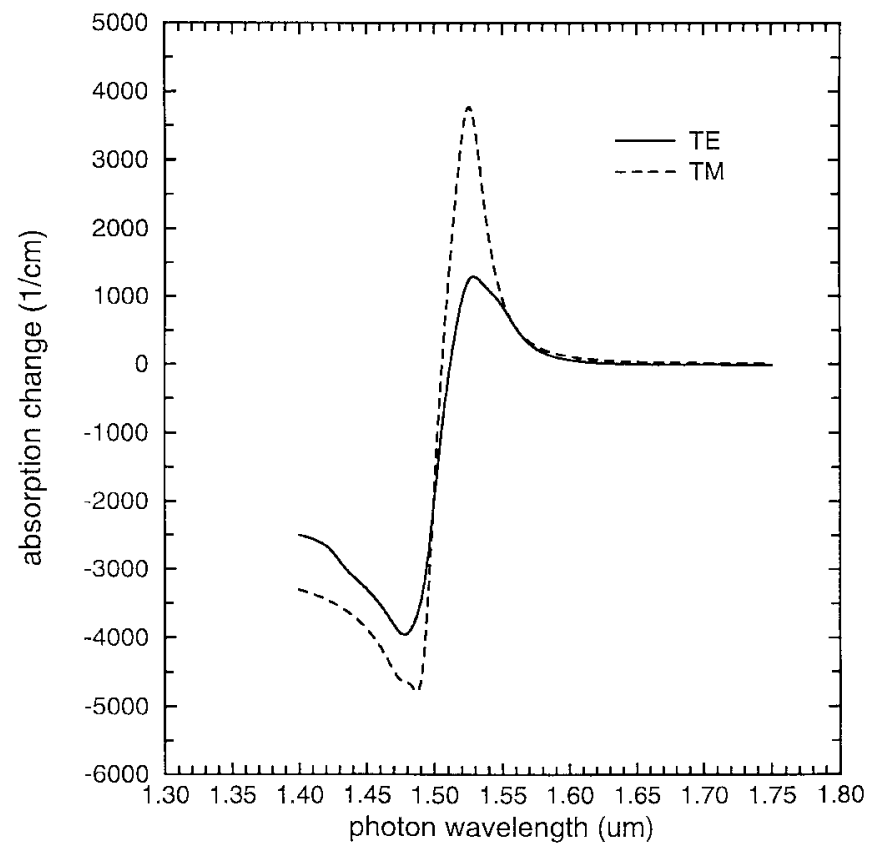

Fig. 8. The TE and TM absorption change spectra of DFQW with In content of 0.53 , well width $12 \mathrm{~nm}$, and annealing of $1 \mathrm{~h}$ with $F=100 \mathrm{kV} / \mathrm{cm}$.

QW's. Interdiffusion on Group V sublattice only is proposed to develop polarization-insensitive QW's. The twophase interdiffusion model entails similar well widths for both the as-grown and the interdiffused QW's, thus reducing the difficulty in determining parameters such as effective well width and broadening factors after interdiffusion. The criteria and steps which can serve as guidelines to develop DFQW's with electroabsorptive polarization insensitivity are also discussed. In order to optimize the adjustable wavelength range 
of interdiffusion in producing the polarization insensitivity, the as-grown wells should preferably contain no phosphorus content. Our results show that the two DFQW structures, $\mathrm{In}_{0.51} \mathrm{Ga}_{0.49} \mathrm{As}-\mathrm{InP}$ as-grown $\mathrm{QW}$ with well width $11 \mathrm{~nm}$ and $0.15 \%$ as-grown tensile strain, as well as lattice-matched $\mathrm{In}_{0.53} \mathrm{Ga}_{0.47} \mathrm{As}-\mathrm{InP}$ as-grown QW with a well width $12 \mathrm{~nm}$, can achieve electroabsorptive polarization insensitivity at the photon wavelength of $\sim 1.55 \mu \mathrm{m}$. It is thus interesting to note that an as-grown lattice-matched rectangular $\mathrm{QW}$, an easily fabricated QW structure, can produce polarization insensitivity by using QW interdiffusion, which should be attractive from a device application point of view.

\section{REFERENCES}

[1] H. Yamamoto, M. Asada, and Y. Suematsu, "Theory of refractive index variation in quantum well structure and related intersectional optical switch," J. Lightwave Technol., vol. 6, pp. 1831-1840, 1988.

[2] K. Wakita, Y. Kawamura, M. Nabeto, and H. Asai, "Long wavelength waveguide multiple quantum well optical modulators," IEEE J. Quantum Electron., vol. QE-23, pp. 2210-2215, 1987.

[3] T. Ido, M. Koizumi, S. Tanaka, M. Suzuki, and H. Inoue, "Polarization and wavelength insensitive MQW electroabsorption optical gates for WDM switch systems," IEEE Photon. Technol. Lett., vol. 8, pp. 788-790, 1996.

[4] K. G. Ravikumar, T. Aizawa, and R. Yamauchi, "Polarizationindependent field-induced absorption-coefficient variation spectrum in an InGaAs/InP tensile-strained quantum well," IEEE Photon. Technol. Lett., vol. 5, pp. 310-312, 1993.

[5] K. Wakita, I. Kotaka, K. I. Yoshino, S. Kondo, and Y. Noguchi, "Polarization-independent electroabsorption modulators using straincompensated InGaAs-InAlAs MQW structures," IEEE Photon. Technol. Lett., vol. 7, pp. 1418-1420, 1995.

[6] T. Yamaguchi, T. Morimoto, K. Akeura, K. Tada, and Y. Nakano, "Polarization-independent waveguide modulator using a novel quantum well with mass-dependent width," IEEE Photon. Technol. Lett., vol. 6, pp. 1442-1444, 1994.

[7] M. Kato, K. Tada, and Y. Nakano, "Wide-wavelength polarizationindependent optical modulator based on tensile-strained quantum well with mass-dependent width," IEEE Photon. Technol. Lett., vol. 8, pp. 785-787, 1996.

[8] J. C. Zucker, K. L. Jones, T. H. Chiu, B. Tell, and K. B. Goebeler, "Strained quantum wells for polarization-independent electrooptic waveguide switches," J. Lightwave Technol., vol. 10, pp. 1926-1930, 1992.

[9] T. Aizawa, K. G. Ravikumar, S. Suzaki, T. Watanabe, and R. Yamanchi, "Polarization-independent quantum-confined Stark effect in an InGaAs/InP tensile-strained quantum well," IEEE J. Quantum Electron., vol. 30, pp. 585-592, 1994.

[10] B. L. Weiss, Ed., "Quantum well mixing for optoelectronics," Opt. Quantum Electron., vol. 23, pp. S799-S994, 1991, special issue.

[11] B. Tell, B. C. Johnson, J. L. Zyskind, J. M. Brown, J. W. Sulhoff, K. F. Brown-Goebeler, B. I. Miller, and U. Koren, "Disordering of InGaAs-InP quantum wells by Si implantation," Appl. Phys. Lett., vol. 52, pp. $1428-1430,1988$.

[12] N. J. Whitehead, W. P. Gillin, I. V. Bradley, B. L. Weiss, and P. Claxton, "Disorder-induced mixing of InGaAs/InP multiple quantum wells by phosphorus implantation for optical waveguides," Semicond. Sci. Technol., vol. 5, pp. 1063-1066, 1990.

[13] C. J. McLean, J. H. Marsh, R. M. Marsh, R. M. De La Rue, A. C. Bryce, B. Garrett, and R. W. Glew, "Layer selective disordering by photoabsorption-induced thermal diffusion in InGaAs/InP based multiquantum well structures," Electron. Lett., vol. 28, pp. 1117-1119, 1992.

[14] J. Oshinowo, J. Dreybrodt, and A. Forchel, "Photoluminescence study of implantation-induced intermixing of $\mathrm{In}_{0.53} \mathrm{Ga}_{0.47} \mathrm{As} / \mathrm{InP}$ single quantum wells by argon ions," J. Appl. Phys., vol. 74, pp. 1983-1986, 1993.

[15] T. Miyazawa, H. Iwamura, and M. Naganuma, "Integrated externalcavity InGaAs/InP lasers using cap-annealing disordering," IEEE Photon. Technol. Lett., vol. 3, pp. 421-423, 1991.

[16] S. S. Rao, W. P. Gillin, and K. P. Homewood, "Interdiffusion of Group III sublattice in In-Ga-As-P/In-Ga-As-P and In-Ga-As/In-Ga-As heterostructures," Phys. Rev. B, vol. 50, pp. 8071-8073, 1994.
[17] A. Hamoudi, A. Ougazzaden, P. Krauz, E. V. K. Rao, M. Jouhel, and $\mathrm{H}$. Thibierge, "Cation interdiffusion in InGaAsP/InGaAsP multiple quantum wells with constant P/As ratio," Appl. Phys. Lett., vol. 66, pp. 718-720, 1995.

[18] B. B. Elenkrig, D. A. Thompson, J. G. Simmons, D. M. Bruce, Y. Si, J. Zhao, J. D. Evans, and I. M. Templeton, "Experimental study of implantation-induced disordering in InGaAsP strained multi-quantum well heterostructure," Appl. Phys. Lett., vol. 65, pp. 1239-1241, 1994.

[19] C. Francis, F. H. Julien, J. Y. Emery, R. Simes, and L. Goldstein, "Selective bandgap blueshifting of InGaAsP/InGaAs(P) quantum wells by thermal intermixing with phosphorus pressure and dielectric capping," J. Appl. Phys., vol. 75, pp. 3607-3610, 1994.

[20] T. Fujii, M. Sugawara, S. Yazaki, and K. Nakajima, "Macroscopic mechanism of Group V interdiffusion in undoped InGaAs/InP quantum wells grown by MOVPE," J. Cryst. Growth, vol. 105, pp. 348-352, 1990.

[21] I. J. Pape, P. Li Kam Wa, J. P. R. David, P. A. Claxton, and P. N. Pobson, "Disordering of $\mathrm{Ga}_{0.47} \mathrm{In}_{0.53} \mathrm{As} / \mathrm{InP}$ multiple quantum well layer by sulphur diffusion," Electron. Lett., vol. 24, pp. 1217-1218, 1988.

[22] K. Mukai, M. Sugawara, and S. Yamazaki, "Interdiffusion process in lattice-matched $\operatorname{In}_{x} \mathrm{Ga}_{1-x} \mathrm{As}_{y} \mathrm{P}_{1-y} / \mathrm{InP}$ and $\mathrm{GaAs} / \mathrm{Al}_{x} \mathrm{Ga}_{1-x}$ As quantum well," Phys. Rev. B, vol. 50, pp. 2273-2282, 1994.

[23] T. P. Pearsall, Ed., GaInAsP Alloy Semiconductors. New York: Wiley, 1982 , p. 295

[24] J. Micallef, E. H. Li, and B. L. Weiss, "Effect of interdiffusion on the sub-band-edge structure of $\operatorname{In}_{0.53} \mathrm{Ga}_{0.47} \mathrm{As} / \mathrm{InP}$ single quantum well," J. Appl. Phys., vol. 73, pp. 7524-7532, 1993.

[25] W. Bloss, "Electronic field dependence of quantum-well eigenstates," $J$. Appl. Phys., vol. 65, pp. 4789-4794, 1989.

[26] T. F. Jiang, "An alternative approach to exciton binding energy in a GaAs/AlGaAs quantum well," Solid State Commun., vol. 50, pp. 589-593, 1984

[27] W.-C. Shiu, J. Micallef, I. Ng, and E. H. Li, "Effects of different cation and anion interdiffusion rates in disordered in ${ }_{0.53} \mathrm{Ga}_{0.47} \mathrm{As} / \mathrm{InP}$ Single Quantum Wells," Jpn. J. Appl. Phys., vol. 34, pp. 1778-1783, 1995.

[28] J. Micallef, J. L. Borg, and E. H. Li, "Interdiffused InGaAs/InP quantum wells for polarization-independent electroabsorption," Opt. Quantum Electron., vol. 29, pp. 423-428, 1997.

Wallace C. H. Choy received the B.Sc. degree in applied physics fromthe Baptist University of Hong Kong in 1994 and the M.Phil. degree in electronic engineering from the University of Hong Kong in 1996. His thesis was concerned with the modeling of optical properties of interdiffusion modified quantum-well structures for modulation devices. He is currently pursuing the $\mathrm{Ph} . \mathrm{D}$. degree in electronic engineering at the University of Surrey, U.K.

He currently holds a Croucher Foundation Scholarship. His main research topics include the influence of acoustic waves in quantum-well materials and acoustooptic devices for optoelectronics applications.

Mr. Choy was awarded the Sir Edward Youde Memorial Fellowship and a Postgraduate Studentship award while performing his graduate work.

E. Herbert Li (S'87-M'88-SM'95), for photograph and biography, see $\mathrm{p}$ 1009 of the June 1997 issue of this JournaL.

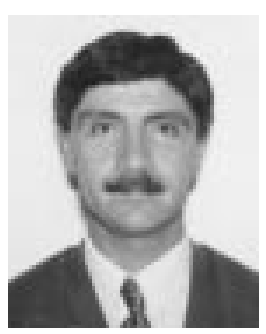

Joseph Micallef (M'92) received the B.Sc.Eng. degree in electronics engineering from the University of Malta, Msida, in 1972 and the M.Sc. and Ph.D degrees in electrical engineering from the University of Surrey, U.K., in 1989 and 1993, respectively.

From 1973 to 1981, he was with General Instruments, engaged in work on high-voltage components and circuits and on IFT's. He moved to SGS-THOMSON Microelectronics in 1981 where he was involved with packaging of MOS IC's. In 1989, he joined the Faculty of Engineering at the University of Malta and is now with the Department of Microelectronics. His current research activities include optical properties of III-V quantum-well structures, as well as IC design. 